\title{
Studies Regarding Treatments of Led-s Emitted Light on Sprouting Fagopyrum Esculentum Moench
}

\author{
Oana LIVADARIU ${ }^{1 *}$, Carmen MAXIMILIAN ${ }^{2}$ \\ ${ }^{1}$ Faculty of Biotechnologies, UASVMB, 59 Bd. Marasti, 011464 Bucharest, Romania \\ ${ }^{2}$ Institute of Biology Bucharest, Romanian Academy, 296 Spl. Independentei, 060031, Bucharest, \\ Romania \\ *Corresponding author, e-mail: ombioteh@gmail.com
}

Bulletin UASVM Animal Science and Biotechnologies 74(2)/ 2017

Print ISSN 1843-5262; Electronic ISSN 1843-536X

DOI:10.15835/buasvmcn-asb: 0008

\begin{abstract}
The present study presents the influence exercised by LED (light emitting diodes)-s emitted white (W), red (R), blue (B) light on sprouting of buckwheat seeds and evaluated the effects on content of phenolic compounds, antioxidant capacity and flavonoids.The fresh weight of hypocotyls, showed that the R light has generated the best influence for sprouts and $\mathrm{W}$ light has generated the most adverse influence. The content of flavonoids was stimulated by R LED for variant sprouts with testa (V3), while both B and R LED inhibited for variant without testa (V4, V6). The higher concentration of polyphenols was obtained for V5 variant illuminated with B LED and increased for V4 variant illuminated with R LED. The antioxidant capacity was higher for V3 variant illuminated with B light and stimulated by R LED in V4 variant.The results contribute to the establishment an efficient working protocol, for obtaining sprouts from buckwheat seeds, with a comprehensive content of bioactive components, various nutrients by applying the R LED treatment.
\end{abstract}

Keywords: buckwheat seed, sprout, LED-s, antioxidant activity, polyphenols, flavonoids

\section{INTRODUCTION}

Fagopyrum esculentum Moench. (buckwheat), of Polygonaceae family and Fagopyrum type (Jing et al., 2016), is a species of an annual herbaceous plant, originating from Tibet, having multiple uses in human food, zootechnical industry, apiculture and human medicine (Pârvu, 1997). This is a species with valuable composition seeds (proteins, food fibers, lipids with unsaturated fats, vitamins, amino acids, antioxidants, phenols, flavonoids - Pârvu, 1997; Alvarez-Jubete et al., 2010; Jing et al., 2016; Park, 2017), for human nutrition. Fagopyrum esculentum Moench. (buckwheat), is also a species naturally gluten-free (Kupper, 2005; Coeliac UK, 2017).

Sprouts of Fagopyrum esculentum Moench. (buckwheat), contain various compounds of a biological activity (rutin, proteins, etc.), which have bio-pharmaceutical uses (Li and Zhang, 2001) as well as high anti-oxidizing activity (Kim et al., 2008).

As a consequence, whereas also that the demand for functional food is gradually increasing, getting sprouts at an experimental research level in sterile conditions can lay the foundation for the extension of getting the same at the microproduction level.

Polyphenols are secondary plant metabolites that play a role in the protection of plants against ultraviolet radiation, pathogens and herbivores (Harborne and Williams, 2000; Alvarez-Jubete et al., 2010). Fruits and beverages, such as tea, red wine and coffee, are the main sources of polyphenols, however, vegetables, cereals or pseudocereals (as buckwheat) and leguminous plants are also good sources.

TheLEDs treatments, appliedforobtain sprouts from seeds were tested at Fagopyrum esculentum (all blue, red:blue:far-red or red:green:blue - Zakir H. M., 2007), and F. tataricum Gaertn. (red, blue or 
red:blue - Sang-Won et al., 2014). Also, the LEDs treatments (red:blue or red:blue:white), were tested for hydroponically grown at Lactuca sativa L. var. capitata (Lin et al., 2013).

The processing can modify the polyphenol content of foods in several ways (Manach et al., 2005). In particular, sprouting has been reported as a means of increasing the polyphenol content of buckwheat seeds (Kim et al., 2004). Conversely, studies have shown that thermal processing of fruits, vegetables and cereals can have a detrimental effect on flavonoid compounds (Dietrych-Szostak and Oleszek, 1999). In this respect, our studies evaluated the content of polyphenols, flavonoids and antioxidant capacity of buckwheat sprouts illuminated with white, red and blue LED.

\section{MATERIALS AND METHODS}

The vegetal biological material consisted of seeds of Fagopyrum esculentum Moench. (buckwheat), as procured out of a trade source and finding themselves in dormancy phase. Seeds have been grouped in two categories, namely: S1 (the category of seeds with testa - IN V1, V3 and V5) and S2 (the one without such testa - in V2, V4 and V6).

The working method, in terms of the vegetal biological material Fagopyrum esculentum Moench., has involved the compliance with the work conditions at the hood and it consisted of the following: seeds aseptization, by means of ethylic alcohol solution $\left(\mathrm{C}_{6} \mathrm{H}_{12} \mathrm{O}_{6}\right)$, for 1'; removing the aseptization solution by three washing sessions with aseptized distilled water $\mathrm{H}_{2} \mathrm{O}$ (Badea and Săndulescu, 2001; Cachiță - Cosma et al., 2004); seeds inoculation on aseptized gauze and located in transparent containers; wetting the gauze with aseptized distilled water $\mathrm{H}_{2} \mathrm{O}$ in a share of $10 \mathrm{ml} /$ upon seeds inoculation and $10 \mathrm{ml} /$ the sixth day as of such seeds inoculation.

The experimental device, as used in testing treatments by LEDs emitted light in order to get sprouts out of Fagopyrum esculentum Moench. seeds was made up of three sets of LEDs (LED = Light Emitting Diode). LEDs have emitted light out of the white, red or blue light spectrum (Zakir, 2007; Lin et al., 2013; Sang-Won et al., 2014), subject to the treatment being applied. The technical specifications of LEDs are: power 18 $\mathrm{W}$, voltage $220 \mathrm{~V}$, light flux $435 \mathrm{~lm}$ and dominant wavelength (cold white, deep red or high blue).
Methods of statistical analysis. Each experimental variant consisted in three repetitions with 45 seeds / repetition. For each individual inoculate were made the measurements. The average value and standard error were determinate per each experimental variant.

The incubation conditions provided for the temperature of $21^{\circ} \mathrm{C} \pm 2^{\circ} \mathrm{C}$, as well as for the enforcement of treatments for 16 hours within 24 $\mathrm{h}$ period of time (Lin et al., 2013). Treatments have been applied after the first $72 \mathrm{~h}$. During the first $72 \mathrm{~h}$, seeds have been kept in the dark.

The experimental plan, as used for testing treatments by LEDs - emitted light in order to get sprouts out of Fagopyrum esculentum Moench. seeds consisted of six experimental variants, respectively:

- V1 / WS1 = treatment by white color LED - emitted light (W) + seeds with testa (S1- control);

- V2 / WS2 = treatment by white color LED emitted light $(W)+$ seeds without testa (S2 control);

- V3 / RS1 = treatment by red color LED - emitted light (R) + seeds with testa (S1);

- V4 / RS2 = treatment by red color LED - emitted light (R) + seeds without testa (S2);

- V5 / BS1 = treatment by blue color LED - emitted light (B) + seeds with testa (S1) and

- V6 / BS2 = treatment by blue color LED - emitted light (B) + seeds without testa (S2).

Each such experimental variant included 45 Fagopyrum esculentum Moench. seeds and it has been repeated three times. For each inoculation one has conducted quantitative determinations (the sprouts rate, the fresh weight of sprouts, the fresh weight of cotyledons, the fresh weight of hypocotyls and the fresh weight of roots), as well as morphometric determinations (the length of hypocotyls).

The biological material of Fagopyrum esculentum Moench. (sprouts with testa - S1 or without testa - S2), has also been analyzed by biochemical determinations (the contents in polyphenol compounds, the antioxidizing capacity and the content of flavonoid).

Preparation of methanolic extracts. $4 \mathrm{ml}$ of $100 \%$ methanol were added to $1 \mathrm{~g}$ of sprout + seed buckwheat and grounded in a mortar with pestle. The extract was maintained overnight to $4^{\circ} \mathrm{C}$. After centrifugation $20 \mathrm{~min}$. at $15.000 \mathrm{rpm}$ 
the supernatant was used for determination of phenolic compounds, antioxidant capacity and flavanoids content.

The polyphenol content of methanolic extracts were evaluated using a modified method of the Folin-Ciocalteu reagent (Mihailović et al., 2013). The reaction mixture consisted from 0.5 $\mathrm{ml}$ methanolic extract, $2.5 \mathrm{ml}$ Folin-Ciocalteu diluted $1: 10$ and $2 \mathrm{ml} 7.5 \% \mathrm{Na}_{2} \mathrm{CO}_{3}$.Ine mixture was incubated for $30 \mathrm{~min}$. at room temperature. The absorbance was measured at $765 \mathrm{~nm}$. The calibration curve was prepar with different concentrations of gallic acid. The results are expressed in mg gallic acid equivalent/g fresh weight.

The antioxidant capacity of methanolic extracts were carried out according to Marxen et al. (2007), using DPPH (2,2-diphenyl-1picrylhydrazyl) and a calibration curve with Trolox as antioxidant standard. The mixture was incubated at room temperature for $30 \mathrm{~min}$. and spectrophotometrically detected at $517 \mathrm{~nm}$. The antioxidant capacity was expressed in mMTrolox/g fresh weight.

The flavonoid compounds of methanolic extracts were estimated using Zhishen et al. (1999), modified method with aluminum chloride. The $0.5 \mathrm{~mL}$ of methanolic extract were mixed with $2 \mathrm{ml}$ of distilled water and $150 \mu \mathrm{l}$ of $5 \%$ sodium nitrate. After $5 \mathrm{~min}$., added $150 \mu \mathrm{l}$ of $10 \%$ aluminum chloride and incubated for $6 \mathrm{~min}$ and then $2 \mathrm{ml}$ of $4 \%$ sodium hydroxide were added. Absorbance of the mixtures was measured at 510 nm. It used a calibration curve with rutin.

The experimental outcomes having been recorded, as per each parameter having been determined, have been statistically analyzed and processed under the form of an average value and standard deviation. The flavonoids concentration was expressed in mg rutin equivalent/g fresh weight.

\section{RESULTS AND DISCUSSIONS}

The experimental outcomes, as got by testing LEDs-emitted light - based treatments in order to get sprouts out of Fagopyrum esculentum Moench. seeds and duly registered in terms of quantitatively determined parameters.

The experimental results, as got for the average values of the sprouts rate (Figure 1), as per experimental variant, show a certain evolution in time, between the fifth day and the eight day, which varies a great deal, subject to the category of the vegetal biological material (S1 or S2) and which does not vary at all, or which is almost similar, subject to the color of the light being used for the enforcement of the irradiation treatment (W, R or B). Thus, in the case of the experimental variants being tested (V1, V3 and V5), in terms of the biological material under category S1, one may notice both the increase of the sprouts rate, as got in the case of white light treatments (from $34 \pm 0.71$ to $37 \pm 0.27 / \mathrm{WS} 1$ ) or blue light (from $34 \pm 0.71$ to $38 \pm 0.28 / \mathrm{BS1}$ ), as well as the stagnation of the sprouts rate, as got in the case of the red light treatment $(38 \pm 0.28 / \mathrm{RS} 1)$. Whereas

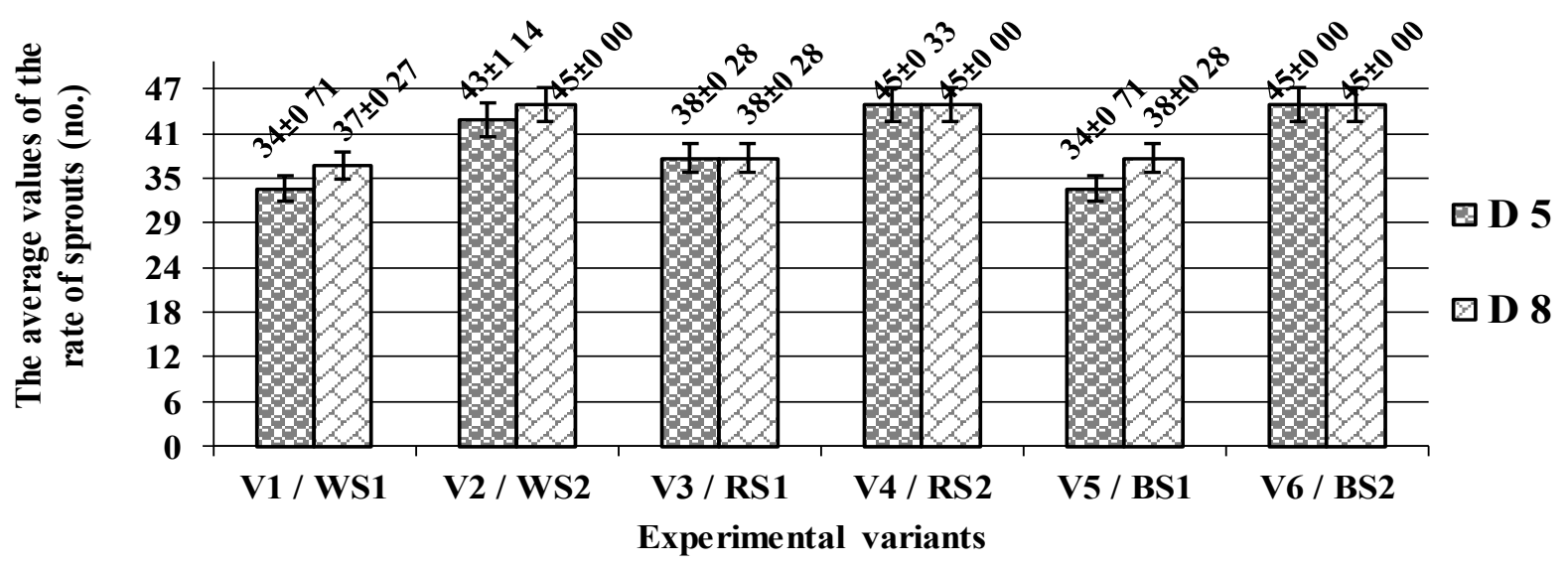

Fig. 1. Evolution in time (days - D5 and D8), in terms of the average values of the rate of sprouts, as got (no.) subject to the experimental variant (V1 / WS1, V2 / WS2, V3 / RS1, V4 / RS2, V5 / BS1 and V6 / BS2) being tested 


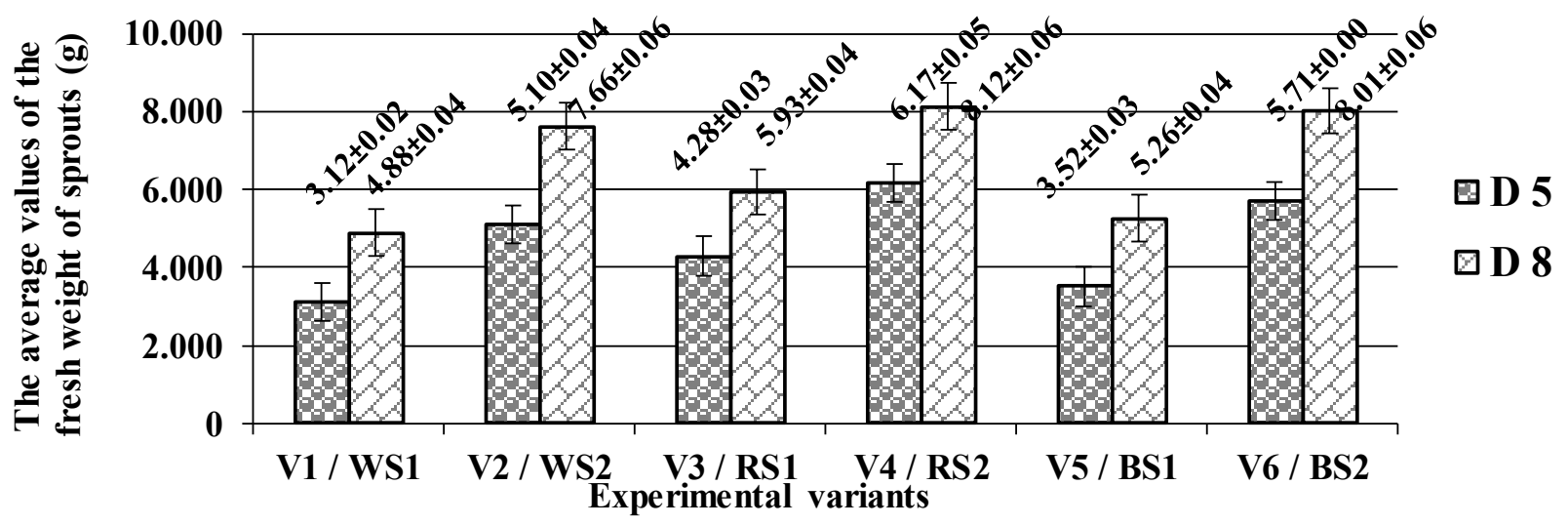

Fig. 2. Evolution in time (days - D5 and D8), in terms of the average values of the overall fresh weight of sprouts (g), subject to the experimental value (V1 / WS1, V2 / WS2, V3 / RS1, V4 / RS2, V5 / BS1 and V6 / BS2) being tested

in the case of the experimental variants being tested (V2, V4 and V6), in terms of the biological material under category S2, one finds an increase of the rate of sprouts, as got only in the case of the treatment by white light (from 43 to 45/WS2), and the maintenance of the rate of sprouts, as used in the case of treatments by red light (45 / RS2) or blue light (45/BS2).

The experimental outcomes, as achieved in terms of the average values of the overall fresh weight of sprouts (Figure 2), per experimental variant being tested, show a certain evolution in time, between the fifth and the eighth day. This evolution is variable and upward both in terms of S1 as well as in terms of S2, showing distinctly significant outcomes and only one insignificant result.

Subject to the color of the light being used for the enforcement of the irradiation treatment (W, R or B), in the case of the biological material under category $\mathrm{S} 1$, one may notice that on the fifth day, there occur a series of very high fluctuations (between V1/WS1 and V3/RS1, as well as between V3/RS1 and V5/) and some high fluctuations (between V1/WS1 and V5/BS1).Whereas, in terms of the eighth day, one may still notice such very high fluctuations (between V1/WS1 and V3/ RS, as well as between V3/RS1 and V5/BS1), and some high such fluctuations (between V1/WS1 and V5/BS1).

Also, subject to the color of the light being used for the enforcement of the irradiation treatment (W, R or B), in the case of the biological material under category S2, one may notice that on the fifth day, there occur just one may notice that on the fifth day, there occur just very high fluctuations (between V2/WS2 and V4/RS2, between V2/WS2 and V6/BS2, and between V4/RS2, and V6/BS2). And on the eighth day one may notice the following types of fluctuations: very high (between V2/WS2 and V4/RS2), high (between V2/WS2 and V6/ BS2), and low (between V4/RS2 and V6/BS2).

The experimental outcomes being achieved in terms of the fresh weight of cotyledons (Figure 3), per experimental variant, show a certain evolution in time, in between the fifth and the eighth day. Such evolution is variable and upward both in terms of S1 and in terms of S2, showing distinctly significant results, insignificant results and only one such significant result.

Subject to the color of the light being used for the enforcement of the irradiation treatment (W, R or $\mathrm{B}$ ), in the case of the biological material under category S1, one may notice that on the fifth day, there occur only very low fluctuations (between V1/WS1 and V3/RS1, between V1/WS1 and V5/ BS1, and between V3/RS1 and V5/BS1). And in terms of the eighth day, one may notice both very high fluctuations (between V1/WS1 and V5/BS1, and between V3/RS1 and V5/BS1), as well as one very low fluctuation (between V1/WS1 and V3/ RS1).

Also, subject to the color of the light being used for the enforcement of the irradiation treatment (W, R or B), in the case of the biological material under category S2, one may notice that on the 


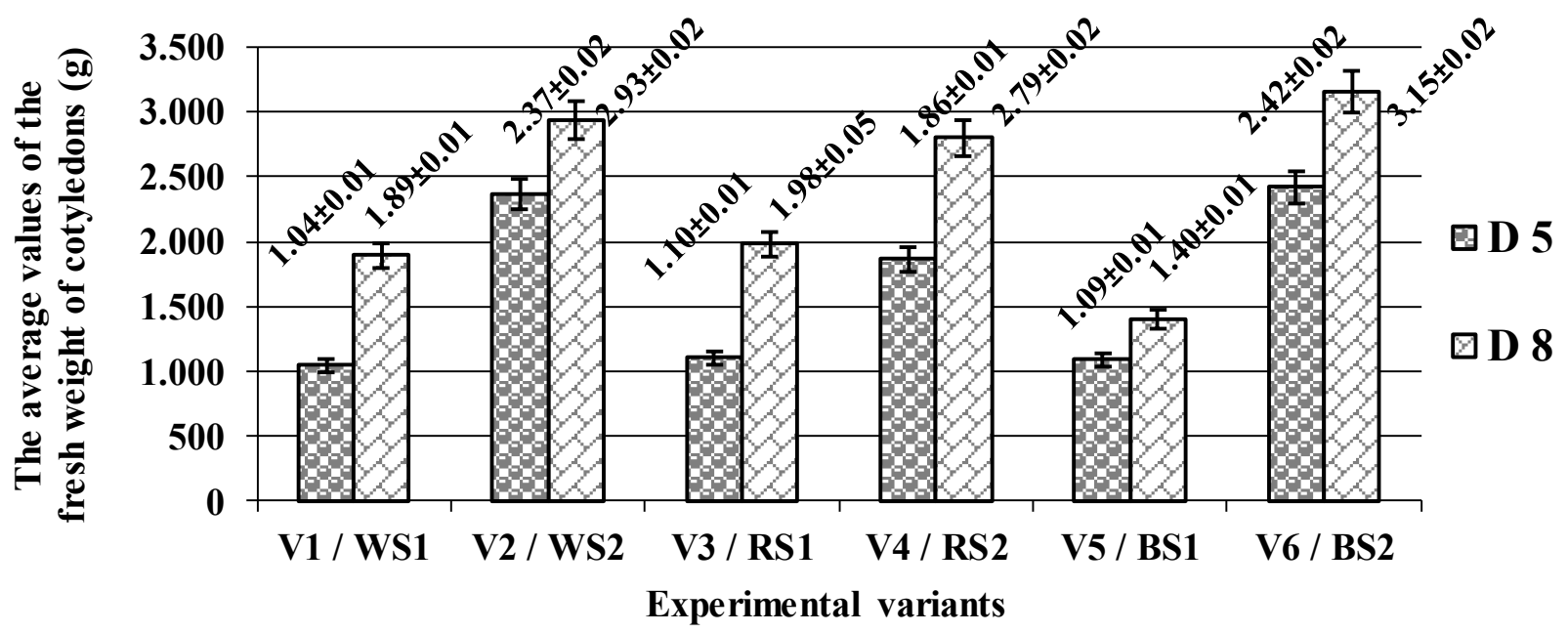

Fig. 3. Evolution in time (days - D5 and D8), in terms of the average values of the fresh weight of cotyledons (g), subject to the experimental variant (V1 / WS1, V2 / WS2, V3 / RS1, V4 / RS2, V5 / BS1 and V6 / BS2) being tested

fifth day, there occur both very high fluctuations (between V2/WS2 and V4/RS2, as well as between V4/RS2 and V6/BS2 in terms of S2), as well as one very low fluctuation (between V2/WS2 and V6/ BS2). Whereas, in terms of the eighth day, one may notice the following types of fluctuations: high (between V4/RS2 and V6/BS2), average (between V2/WS2 and V6/BS2), and low (between V2/WS2 and V4/RS2).

The experimental outcomes being achieved in terms of the average values of the fresh weight of hypocotyls (Figure 4), per experimental variant being tested, show a certain evolution in time, in between the fifth and the eighth day. Such evolution is variable and upward both in terms of S1 and in terms of S2, showing distinctly significant and significant results.

Subject to the color of the light being used for the enforcement of the irradiation treatment (W, R or $\mathrm{B}$ ), in the case of the biological material under category S1, one may notice that on the fifth day, there occur both very high fluctuations (between V1/WS1 and V3/RS1, as well as between V3/ RS1 and V5/BS1), as well as one low fluctuation (between V1/WS1 and V5/BS1). Whereas, in terms of the eighth day, one may notice the following types of fluctuations: very high (in between V1/ WS1 and V3/RS1), high (in between V1/WS1 and V5/BS1), and low (in between V3/RS1 and V5/ BS1).

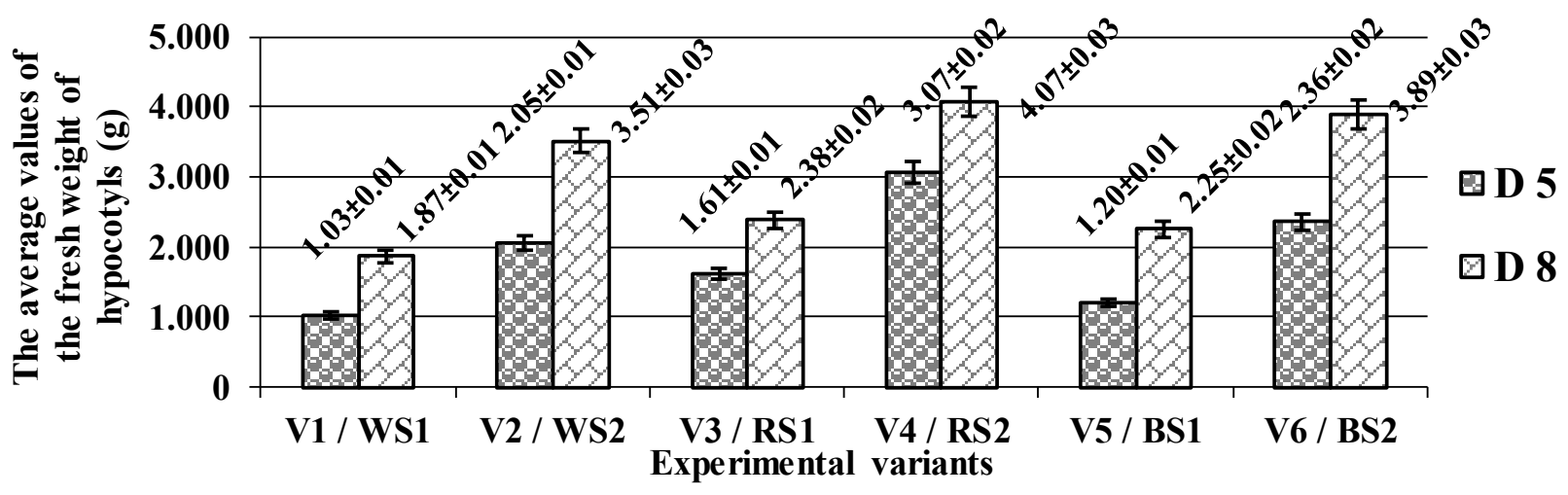

Fig. 4. Evolution in time (days - D5 and D8), in terms of the average values of the fresh weight of hypocotyls (g), subject to the experimental variant (V1 / WS1, V2 / WS2, V3 / RS1, V4 / RS2, V5 / BS1 and V6 / BS2) being tested 
Also, subject to the color of the light being used for the enforcement of the irradiation treatment (W, R or B), in the case of the biological material under category S2, one may notice that on the fifth day, there occur the following types of fluctuations: very high (in between V2/WS2 and V4/RS2), high (between V2/WS2 and V6/ BS2), and low (between V4/RS2 and V6/BS2). And in terms of the eighth day, one may notice the following fluctuations: very high (in between V2/ RS2 and V4/BS2), high (in between V2/WS2 and V6/BS2), and low (in between V4/WS2 and V6/ RS2).

The experimental outcomes duly achieved in terms of the average values of the fresh weight of roots (Figure 5), per experimental variant being tested, show a certain evolution in time, in between the fifth and the eighth day. Such evolution is variable and upward both in terms of $\mathrm{S} 1$, as well as in terms of $\mathrm{S} 2$, showing insignificant, significant results and only one distinctly significant result.

Subject to the color of the light being used for the enforcement of the irradiation treatment (W, R or B), in the case of the biological material under category $\mathrm{S} 1$, one may notice that on the fifth day, there occur both very low fluctuations (in between V1/WS1 and V5/BS1, as well as between V3/RS1 and V5/BS1), as well as one low fluctuation (in between V1/WS1 and V3/ RS1). Whereas, on the eight day, one may notice only low such fluctuations (V1/WS1 and V3/RS1, between V1/WS1 and V5/BS1, as well as between
V3/RS1 and V5/BS1). Also, subject to the color of the light being used for the enforcement of the irradiation treatment (W, R or B), in the case of the biological material under category S2, one may notice that on the fifth day, there occur both low fluctuations (in between V2/WS2 and V6/BS2, as well as between V4/RS2 and V6/BS2), as well as one average fluctuation (between V2/WS2 and V4/RS2).Whereas, in terms of the eighth day, one may notice both average fluctuations (between V2/WS2 and V6/BS2, as well as between V4/RS2 and V6/BS2), as well as one very high fluctuation (between V2/WS2 and V4/RS2).

The experimental outcomes, as got by testing LEDs-emitted light - based treatments in order to get sprouts and duly registered in terms of morphometrically determined parameters.

The experimental results duly registered in terms of the average values of the length of hypocotyls (Figure 6), as achieved from Fagopyrum esculentum Moench. sprouts, per experimental value, show a certain evolution in time, in between the fifth and the eighth day, which varies in a highly significant degree, subject to the category of vegetal biological material (S1 or S2), and which varies quite significantly, subject to the color of the light used for enforcing the relevant irradiation system (W, R or B).

As a consequence, the best experimental results in terms of S1, have been achieved following the treatment by LEDs-red light emission (V3), since the average values of the length of hypocotyls

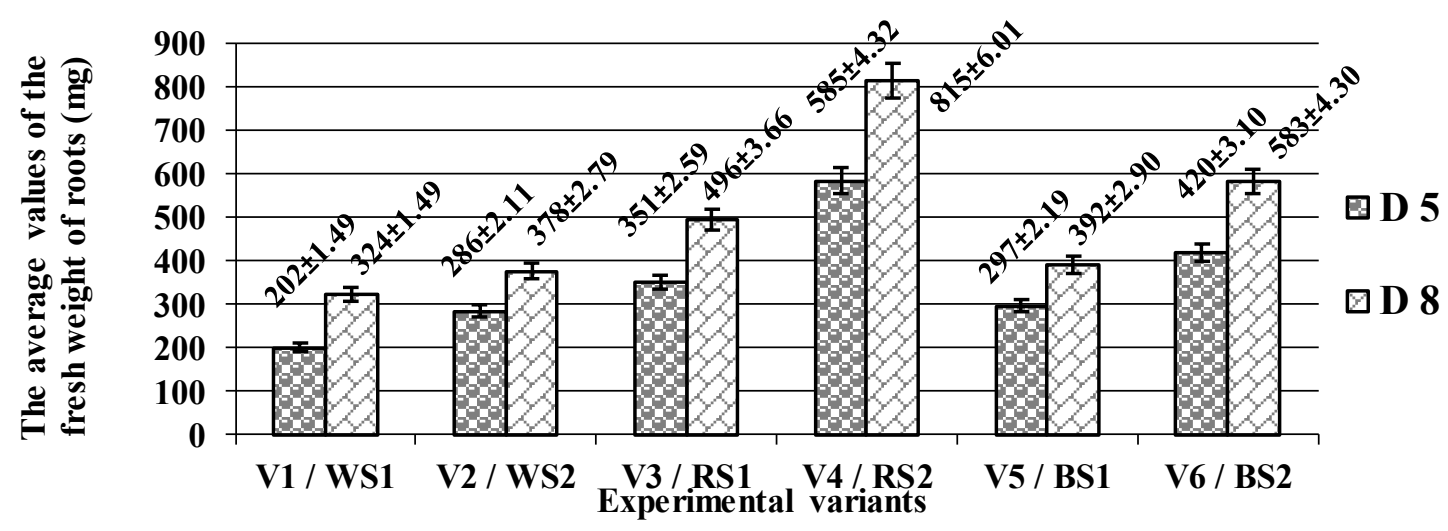

Fig. 5. Evolution in time (days - D5 and D8), in terms of the average values of the fresh weight of roots (mg), subject to the experimental variant (V1 / WS1, V2 / WS2, V3 / RS1, V4 / RS2, V5 / BS1 and V6 / BS2) being tested 


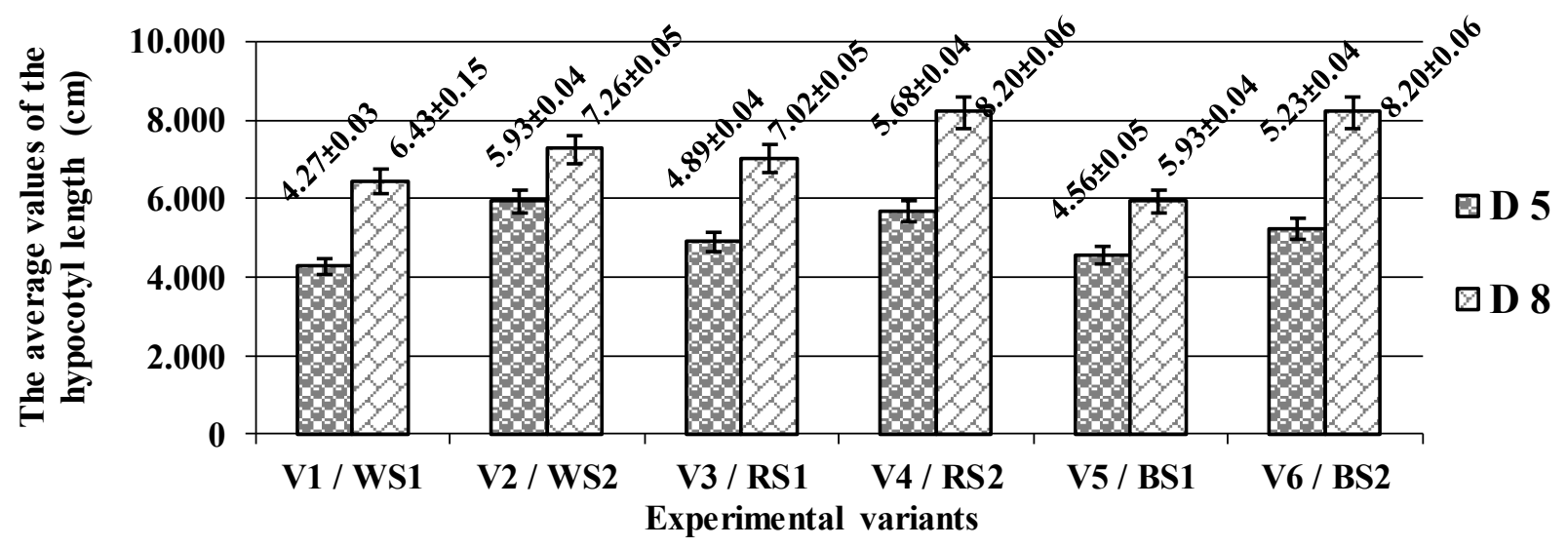

Fig. 6. Evolution in time (days - D5 and D8), in terms of the average values of the length of hypocotyls $(\mathrm{cm})$, as achieved from Fagopyrum esculentum Moench. sprouts, subject to the experimental variant (V1 / WS1, V2 / WS2, V3 / RS1, V4 / RS2, V5 / BS1 and V6 / BS2) being tested

from Sprouts have reached their maximum level, both on the fifth day (V3 by $4.89 \mathrm{~cm} / \mathrm{WS} 1$ ), as well as on the eighth day (V3 by $7.02 \mathrm{~cm} / \mathrm{WS} 1$ ). In terms of $\mathrm{S} 2$, the white light based treatment has triggered the best experimental outcomes in terms of the fifth day (V2 by $5.93 \mathrm{~cm} / \mathrm{WS} 2$ ). Also in terms of $\mathrm{S} 2$, treatments by red or blue light have triggered the best experimental results as related to the eighth day (V4 by $8.20 \mathrm{~cm} / \mathrm{RS} 2$ and V6 again by $8.20 \mathrm{~cm} / \mathrm{BS} 2)$.

Whereas, the lowest experimental results, in terms of S1, have been achieved by the treatments accomplished by LEDs-white or blue light emission (V1 or V5), since the average values of hypocotyls from sprouts have reached their minimum level, on the fifth day (V1 by $4.27 \mathrm{~cm} / \mathrm{WS} 1$ ) or on the eighth day (V5 by $5.93 \mathrm{~cm} / \mathrm{BS} 1$ ). And in terms of S2, treatments applied by blue or white light (V6 or V2), have triggered the lowest experimental outcomes, since the average values of the length of hypocotyls have reached their minimum level, both in terms of the fifth day (V6 by $5.23 \mathrm{~cm}$ / BS2), as well as in terms of the eighth day (V2 by $7.26 \mathrm{~cm} / \mathrm{WS} 2$ ).

The experimental outcomes, as got by testing LEDs-emitted light - based treatments in order to get sprouts and duly registered in terms of biochemically determined parameters.

The polyphenol content (Figure 7), of sprouts buckwheat illumintated with red and blue varied for variant with (V3/RS1 and V5/BS1) or without (V4/RS2 and V6/BS2) testa. The concentration of polyphenol was inhibited by illumination with red (V3/RS1) light LED while the blue (V5/BS1) light LED amplified for the variant with testa. The illumination with red (V4/RS2) light LED induced a high concentration of polyphenols comparative with control (V2/WS2). The blue (V6/BS2) light

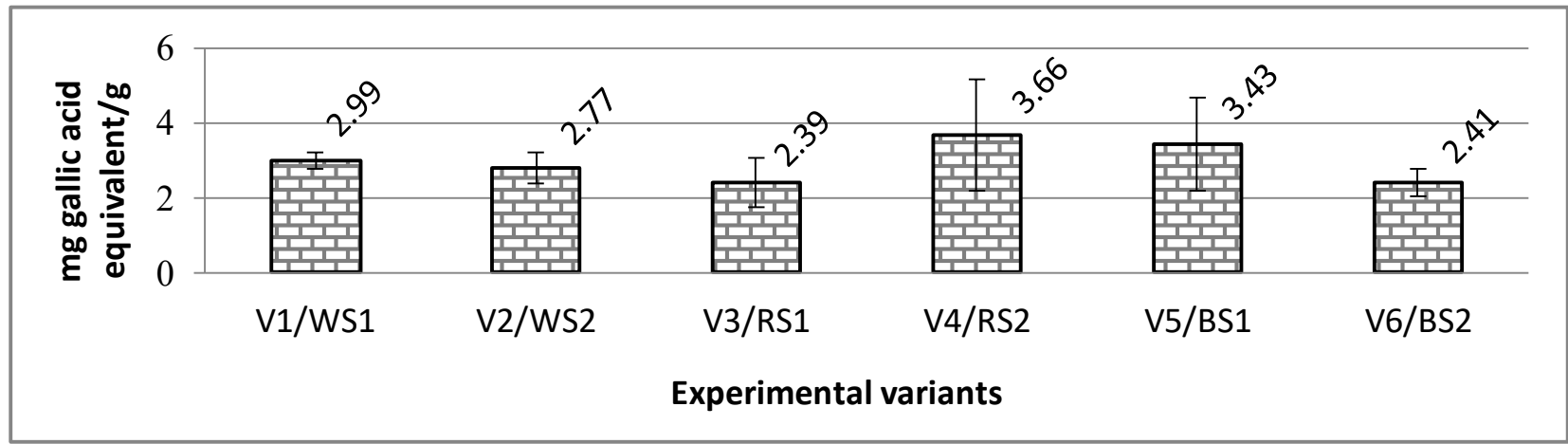

Fig. 7. The polyphenol content of Fagopyrum esculentum Moench. sprouts, subject to the experimental variant (V1 / WS1, V2 / WS2, V3 / RS1, V4 / RS2, V5 / BS1 and V6 / BS2) being tested 
LED induced an inhibition of plyphenol content for the variant without testa.

The antioxidant capacity (Figure 8), was higher for the variant with testa illuminated with blue LED light (V5), comparison with red and white LED light. The red LED light induced the higher value of antioxidant capacity for the variant without testa.

The flavonoid content (Figure 9), was higher for the variant of sprouts iluminated with red (V3/ RS1) LED light (with testa). The blue (V5/BS1) light LED induced an inmhibition of concentration of flavonoids for the variant with testa. The flavonoid concentration was lower for sprouts illuminated with red (V4/RS2) and blue (V6/BS2) LED in comparison with the control (V2/WS2) for the variant without testa (S2 - V2, V4 and V6).

Our results are according with previous reports of Sang-Won et al., 2014 who indicate that the level of total phenolic compound in buckwheat (Fagopyrum esculentum Moench.), sprouts increased by application of red LED for the variant without testa. This is explain by acceleration of polyphenols biosynthesis in the absence of testa and a reduced time for obtaining sprouts.

\section{CONCLUSIONS}

The best experimental results achieved by testing LEDs-emitted white, red or blue light, in order to get sprouts out of Fagopyrum esculentum Moench. seeds and duly registered in terms of the quantitatively determined parameters, regardless of the category of the relevant vegetal biological material (either S1 or S2), have been duly monitored in the case of the treatment by LEDsemitted red light (V3 and V4), since the average values of:

- the rate of sprouts as achieved, have been maximum and similar, in between the fifth and the eighth day (V3 by 38/RS1 and V4 by 45/ RS2);

- the overall fresh weight of sprouts, have been maximum, both in terms of the fifth day (V3 by $4.283 \mathrm{~g} / \mathrm{RS} 1$ and V4 by 6.167g / RS2), as well as in terms of the eighth day (V3 by 5.933g / RS1 and V4 by $8.122 \mathrm{~g} / \mathrm{RS} 2$ );

- the fresh weight of cotyledons, as achieved from sprouts have been maximum, both in terms of the fifth day (V3 by 4.283g /RS1 and V4 by $6.167 \mathrm{~g} / \mathrm{RS} 2)$, as well as in terms of the eighth day (V3 by 5.933g /RS1 and V4 by $8.122 \mathrm{~g} / \mathrm{RS} 2$ );

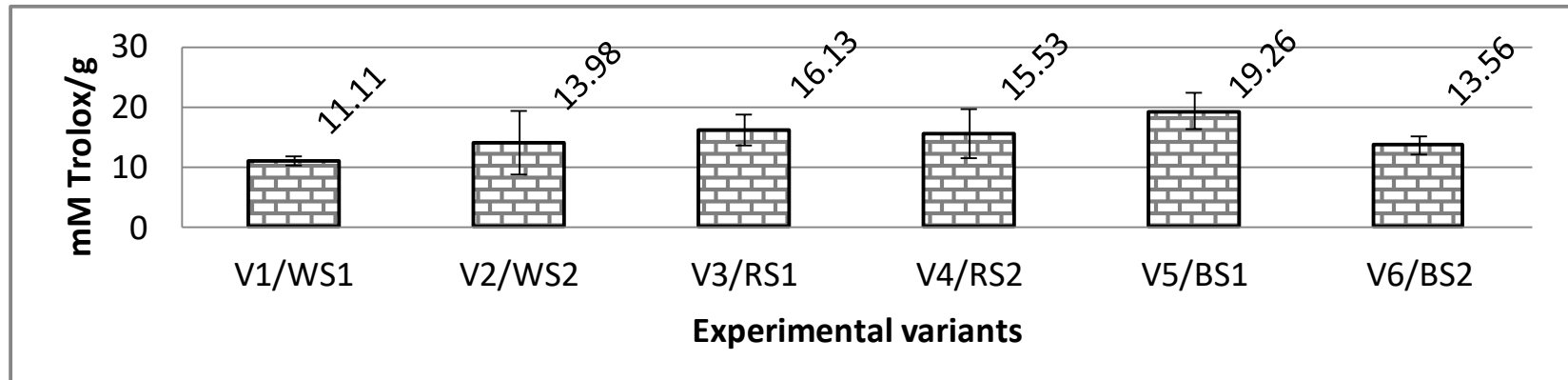

Fig. 8. The antioxidant capacity of Fagopyrum esculentum Moench. sprouts, subject to the experimental variant (V1 / WS1, V2 / WS2, V3 / RS1, V4 / RS2, V5 / BS1 and V6 / BS2) being tested

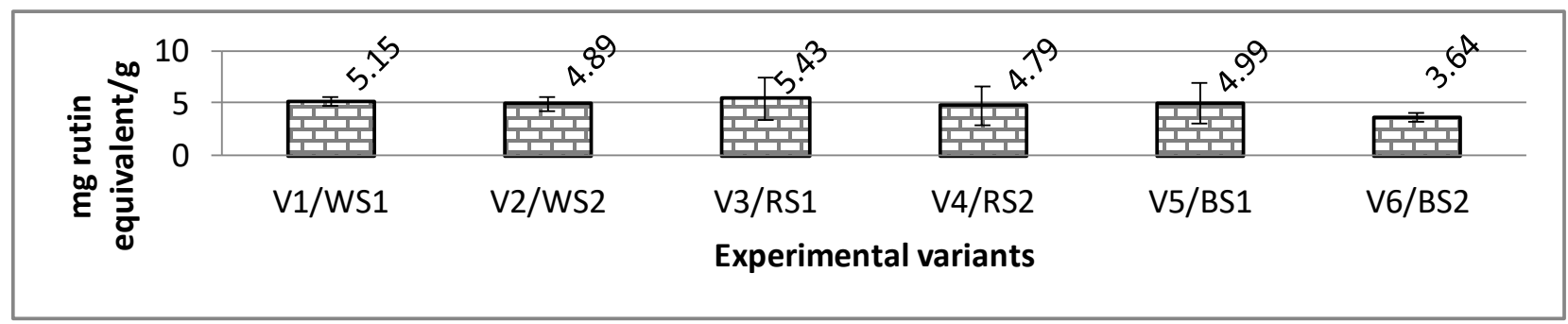

Fig. 9. The flavonoid content of Fagopyrum esculentum Moench. sprouts, subject to the experimental variant (V1 / WS1, V2 / WS2, V3 / RS1, V4 / RS2, V5 / BS1 and V6 / BS2) being tested 
- the fresh weight of hypocotyls as achieved from sprouts have been maximum, both in terms of the fifth day (V3 by $1.611 \mathrm{~g} / \mathrm{RS} 1$ and V4 by $3.070 \mathrm{~g} / \mathrm{RS} 2)$, as well as in terms of the eighth day (V3 by $2.382 \mathrm{~g} / \mathrm{RS} 1$ and V4 by $4.070 \mathrm{~g} / \mathrm{RS} 2$ ) and

- the fresh weight of roots, as achieved from sprouts have been maximum, both in terms of the fifth day (V3 by $351 \mathrm{mg} / \mathrm{RS} 1$ and V4 by $585 \mathrm{mg} / \mathrm{RS} 2)$, as well as in terms of the eighth day (V3 by $496 \mathrm{mg} / \mathrm{RS} 1$ and V4 by $815 \mathrm{mg} / \mathrm{RS} 2$ ).

The best experimental results achieved by testing LEDs-emitted white, red or blue light, in order to get sprouts out of Fagopyrum esculentum Moench. seeds and duly registered in terms of the morphometrically determined parameters, as related to $\mathrm{S} 1$, have been achieved following the treatment by LEDs-emitted red light (V3), since the average values of the length of hypocotyls have reached their maximum level, both on the fifth day (V3 by $4.89 \mathrm{~cm} / \mathrm{WS} 1$ ), as well as on the eighth day (V3 by $7.02 \mathrm{~cm} / \mathrm{WS} 1$ ).Whereas, in terms of $\mathrm{S} 2$, the treatment by white light has triggered the best experimental outcomes on the fifth day (V2 by $5.93 \mathrm{~cm} / \mathrm{WS} 2$ ). And the treatments by red or blue light have triggered the best experimental outcomes in terms of the eighth day (V4 by $8.20 \mathrm{~cm} / \mathrm{RS} 2$ and $\mathrm{V} 6$ again by $8.20 \mathrm{~cm} / \mathrm{BS} 2$ ).

The evaluation of concentrations of polyphenols and flavonoids provides information nutritional quality of buckwheat sprouts. The antioxidant activity higher induced by regarding the effect of blue and red light LED useful for knowing some indicators about the illumination with blue and red light LED is important because the sprouts of pseudocereals represents achievable ingredients in gluten-free bakery products or consumed as raw material for improvement nutritional quality. These light LED tratments can enhance the nutritional quality of buckwheat sprouts and finally the human food.

\section{REFERENCES}

1. Alvarez-Jubete L, Wijngaard H, Arendt EK, Gallagher E (2010). Polyphenol composition and in vitro antioxidant activity of amaranth, quinoa, buckwheat and wheat as affected by sprouting and baking. Food Chemistry, 119, 770-778.

2. Badea EM, Săndulescu D (2001). Bio Tehnologii Vegetale. Editura Fundația Biotech, pp. 21 București.
3. Cachiță-Cosma D, Deliu C, Rakosy-Tican L, Ardelean A (2004). Tratat de biotehnologie vegetală, Ed. Dacia, pp. 105, Cluj-Napoca, Vol. I.

4. Coeliac UK. Grains. (2017). https://www.coeliac.org.uk/ gluten-free-diet-and-lifestyle/gf-diet/grains/ Accessed 09.03.2017.

5. Dietrych-Szostak D, Oleszek W (1999). Effect of Processing on the Flavonoid Content in Buckwheat (Fagopyrum esculentum Moench) Grain. Journal of Agricultural and Food Chemistry, 47(10), 4384-4387.

6. Jing R, Li HQ Hu CL, Jiang YP, Qin LP, Zheng CJ (2016). Phytochemical and Pharmacological Profiles of Three Fagopyrum Buckwheats. International Journal of Molecular Sciences, 17, 4, 589. http://doi.org/10.3390/ ijms17040589/ Accessed 09.03.2017.

7. Harborne JB, Williams CA (2000). Advances in flavonoid research since 1992. Phytochemistry, 55, 481-504.

8. Kim SJ, Zaidul IS, Suzuki T, Mukasa Y, Hashimoto N, Takigawa S, Noda T, Matsuura-Endo C, Yamauchi H (2008). Comparison of phenolic compositions between common and tartary buckwheat (Fagopyrum) sprouts. Food Chemistry, 110, 4, 814-20. doi: 10.1016/j. foodchem.2008.02.050. /Accessed 09.03.2017.

9. Kupper C (2005). Dietary guidelines and implementation for celiac disease. Gastroenterology, 128(4), Supplement 1, 121-127.

10. Kim SL, Kim SK, Park CH (2004). Introduction and nutritional evaluation of bucwheat sprouts as a new vegetable. Food Research International, 37, 319-327.

11. Li SQ, Zhang QH (2001). Advances in the development of functional foods from buckwheat. Crit. Rev. Food Sci. Nutr., 41, 451-464.

12. Lin KH, Huang MY, Huang WD, Hsu MH, Yang ZW, Yang CM (2013). The effects of red, blue and white light-emitting diodes (LEDs) on growth, development and edible quality of hydroponically grown lettuce (Lactuca sativa L. var. capitata). Scientia Horticulturae, 150, 86-91.

13. Manach C, Williamson G, Morand C, Scalbert A, Remesy C (2005). Bioavailability and bioefficacy of polyphenols in humans. I. Review of 97 bioavailability studies. American Journal of Clinical Nutrition, 81(1), 230S-242S.

14. Marxen K, Vanselow KH, Lippemeier S, Hintze R, Ruser A, Hansen UP (2007). Determination of DPPH radical oxidation caused by methanolic extracts of some Microalgal Species by linear regression analysis of spectrophotometric measurements. Sensors, 7, 20802095.

15. Mihailović V, Matić S, Mišić D, Solujić S, Stanić S, Katanić JM, Stanković N (2013). Chemical composition, antioxidant and antigenotoxic activities of different fractions of Gentiana asclepiadea L. roots extract. EXCLI Journal, 12, 807-823.

16. Park CH, Yeo HJ, Park YJ, Morgan AMA, Valan Arasu M, Al-Dhabi NA, Park SU (2017). Influence of Indole-3Acetic Acid and Gibberellic Acid on Phenylpropanoid Accumulation in Common Buckwheat (Fagopyrum esculentum Moench.) Sprouts. Molecules, 22, 374. http:// www.mdpi.com/1420-3049/22/3/374/htm / Accessed 09.03.2017. 
17. Pârvu C (1997). Universul plantelor, Mică enciclopedie. Ed. Enciclopedică, pp. 282-283, Hrișcă (Fagopyrum esculentum), București.

18. Sang-Won L, Jeong MS, Min Ki L, Jin-Hyuk C, Paulrayer A, Mariadhas VA, Tatsuro S, Naif A, Sun-Ju Kimb (2014). Influence of different LED lamps on the production of phenolic compounds in common and Tartary buckwheat sprouts. Industrial Crops and Products, 54, 320-326.
19. Zakir HM (2007). Light emitting diodes increase phenolics of buckwheat (Fagopyrum esculentum) sprouts. Journal of Plant Interaction, 2(1), 71-78. DOI:10.1080/17429140701288228

20. Zhishen J, Mengcheng T, Jianming W (1999). The determination of flavonoid contents in mulberry and their scavenging effects on superoxide radicals. Food Chemistry, 64, 555-559. 